\title{
Effets combinés des dates de semis et de la fertilisation minérale sur la performance du riz NERICA L56 dans la plaine des Mbo, Cameroun
}

\author{
NGOUCHEME Mamouda1*), TABI Fritz Oben11), LONTSI MELI Gilles Raoul'1), FOUADOU Jean²) \\ 1) Département des Sciences du Sol, Faculté d'Agronomie et des Sciences Agricoles, Université de Dschang, BP 222 \\ Dschang, Cameroun. \\ 2) Institut de la Recherche Agricole pour le Développement, BP 44 Dschang, Cameroun. \\ *) Adresse de l'auteur correspondant : Email : ngouch2004@yahoo.fr
}

Original submitted in on $8^{\text {th }}$ January 2020. Published online at www.m.elewa.org/journals/ on $30^{\text {th }}$ April 2020 https://doi.org/10.35759/JABs.148.3

\section{RÉSUMÉ}

Objectifs: L'autosuffisance du Cameroun en riz n'est que de 19\%. Comme conséquence, le pays est dépendant des importations pour satisfaire sa forte demande en cet aliment stratégique. L'indépendance du pays en riz se fera donc par une augmentation de la production à l'hectare. Dans cette optique, la présente étude s'intéresse donc aux performances d'une variété améliorée du riz (NERICA L56) en considérant les dates de semis et les doses d'engrais.

Méthodologie et résultats : Deux essais ont été mis en place à Santchou pendant la saison pluvieuse, à deux dates de semis décalées d'au moins 14 jours (respectivement troisième et première décades de Juillet et Août) en 2017 et répétés en 2018. Les doses d'engrais étaient des combinaisons d'engrais de fond NPK 20-10-10 à deux doses (0 et $300 \mathrm{Kg} / \mathrm{ha}$ ) et d'engrais de couverture (Urée) à cinq doses d'azote $(0,30,60,90$ et $120 \mathrm{Kg} / \mathrm{ha})$. Ces traitements ont été disposés suivant un dispositif en split plot en trois répétitions. Les observations ont été faites à la récolte sur la hauteur des plants, le nombre de talles et de panicules par mètre carré, le nombre total des grains pleins et vides par panicule, le poids de mille grains (PMG), la fertilité des talles, la stérilité des panicules et les rendements en pailles et en grains de paddy. Les effets des traitements ont montré des différences significatives $(p<0,001)$ pour la hauteur des plants, le nombre de talles et panicules au mètre carré, l'infertilité des talles, la stérilité des panicules, les rendements en grains et pailles. Cependant, seules les dates de semis ont eu des effets significatifs $(p<0,001)$ sur le Poids de Mille Grains. Les Rendements Moyens en Riz Paddy (RMRP) sur les deux années d'essai croissaient avec les doses d'engrais de 1,81 à 9,98 t/ha (Semis 1) et de 0,66 à 6,10 t/ha (Semis 2). Les RMRP sur les deux années d'essai diminuaient de 8,20 à 4,55 t/ha du premier au second semis.

Conclusion et application des résultats: Les performances agronomiques du NERICA L56 étaient croissantes avec les combinaisons d'engrais quelle que soit la date de semis. La meilleure date de semis était la troisième décade de Juillet. II serait donc judicieux d'utiliser pour la riziculture pluviale, la formulation NPK : 180 - 30 - 30 (combinaison de 300 Kg NPK 20-10-10 et 120 Kg d'azote sous forme d'urée) pour un semis à la troisième décade de Juillet afin d'obtenir une meilleure performance agronomique du NERICA L56 dans la plaine des Mbo. Toutefois, les autres pratiques culturales (densité de semis, lutte aux 
adventices, entre autres) et le coût d'utilisation d'engrais ne doivent pas être ignorés car les écarts de performance peuvent leur être amputés.

Mots-clés : Dates de semis, Azote, Plaine des Mbo, Nerica L56, Cameroun

\section{ABSTRACT}

Objectives: Cameroon's self-sufficiency in rice is only 19\%. As such, Cameroon greatly depends on importation in order to satisfy her strong domestic demand for this important foodstuff. The country's independence in rice will therefore be achieved by an increase the production per hectare. In this regard, this study aimed to determine the performance of the improved rice variety NERICA L56, taking into consideration the sowing dates and fertilizer combinations.

Methodology and results: Two experimental trials were conducted in Santchou during the rainy season, at two sowing dates of at least 14 days apart (third and first decades of July and August, respectively) in 2017 and repeated in 2018. The fertilizer combinations were NPK 20-10-10 with two levels (0 and $300 \mathrm{Kg} / \mathrm{ha}$ ) and urea with five levels of nitrogen $(\mathrm{N})(0,30,60,90$ and $120 \mathrm{Kg} / \mathrm{ha})$. These treatments were applied following a split-plot design with three replications. The observations were made at the harvest on the rice height, total number of tillers and panicles per meter square, total number of filled and unfilled seeds per panicle, weight of 1000 filled grains, fertility of tiller, sterility of panicles, yields of grain and straw. The effects of these treatments had significant differences $(p<0.001)$ on plant height, number of tillers and panicles per square meter, infertility of tiller, sterility of panicle, yields of grains and straw. However, only the sowing dates had significant effects $(p<0.001)$ on Weight of 1000 filled Grains. The average yields of paddy during the two years experimentation increased with increasing levels of fertilizer from 1.81 to 9.98 t/ha (sowing 1) and from 0.66 to 6.10 t/ha (sowing 2). These average yields decreased from 8.20 to 4.55 tha from the first to second sowing.

Conclusion and applications: The agronomic performances of NERICA L56improved with the combinations of fertilizer, irrespective of the sowing date. The best sowing date was the third decade of July. For good agronomic performance of rice NERICA L56 in the Mbo plain, it is better to use rainfed production, with fertilizer formulation of NPK : $180-30$ - 30 (combination of $300 \mathrm{Kg} \mathrm{NPK} \mathrm{20-10-10} \mathrm{and} 120 \mathrm{Kg}$ of Nitrogen in form of urea) sown at the third decade of July. However, other cultural practices (sowing density, weed control, among others) and cost of fertilizer utilisation must not be ignored because differences in performance can be attributed to them.

Key words: Sowing date, Nitrogen, Mbo plain, NERICA L56 and Cameroon.

\section{INTRODUCTION}

Le Cameroun est un pays à économie typiquement agraire car $70 \%$ d'emplois sont pourvus par l'agriculture (Molua, 2010). Hormis des grandes plantations industrielles et quelques fermes privées, les petits exploitants familiaux dominent l'agriculture au Cameroun (MINADER, 2012). Ces exploitations de subsistance doivent donc être la cible première pour des actions devant faire reculer irréversiblement la pauvreté. Parmi les principales cultures de rente se trouve le riz, qui a un potentiel élevé pour augmenter les revenus des agriculteurs camerounais. Le riz, tout comme le blé et le maïs, occupe une place stratégique et prioritaire dans la sécurité alimentaire au monde.
En Afrique, sa consommation est plus élevée que pour tout autre produit de base. Les causes de cette consommation élevée sont entre autres l'augmentation rapide de la population (3\%/an) et l'évolution des habitudes alimentaires (Seck et al., 2013). Au Cameroun, le riz est largement produit par des petits exploitants de subsistance (Molua, 2010 et Macaulay et Ramadjita, 2015). La production du riz en Afrique en général et au Cameroun en particulier, n'a jamais égalé la forte demande. L'autosuffisance du Cameroun en riz n'est que de $19 \%$ (Tabi et al., 2013). En conséquence, des crises alimentaires (exemple: en 2008) ont été vécues, en plus de la 
dépendance aux importations (Goufo, 2008). Le riz est une culture qui s'adapte à diverses conditions et défis de production. Les sols sur lesquels le riz est cultivé sont aussi variés que les régimes climatiques auxquels les cultures sont exposées (De Dalta, 1981). Compte tenu du régime hydrique, il existe deux types extrêmes de riziculture: la riziculture pluviale stricte et la riziculture irriguée. La riziculture pluviale stricte est alimentée exclusivement par les eaux des pluies (Dalta, 1981; ADRAO, 2008). La riziculture irriguée est alimentée par les eaux d'irrigation avec un régime d'inondation parfaitement maîtrisé et des aménagements hydro-agricoles soignés et constamment entretenus (Dalta, 1981; ADRAO, 2008). La production nationale du riz est estimée à 84000 tonnes/an sur 40000 ha, soit 2,1 tonne/ha. Une bonne partie de cette production estimée à 15000 tonnes est assurée par des paysans dans les bas-fonds et en culture pluviale et le reste des grands périmètres rizicoles (SEMRY, UNDVA, LAGDO) (MINADER, 2012). Les agro systèmes pluviaux des bas-fonds ont souvent plusieurs contraintes à la production du riz telles que la mauvaise qualité du sol, les problèmes de gestion de l'eau, les maladies et les pratiques culturales inapproprié (utilisation d'engrais, date de semis, lutte aux adventices, entre autres.) (Jones, 1995). La dégradation et la déplétion en nutriment ont graduellement augmentées et sont devenues des sérieuses menaces pour la productivité agricole en Afrique (Vanlauve et al., 2002). Les rendements en riz peuvent donc être augmentés avec une application adéquate des engrais chimiques et organiques. Cependant, ceci n'est possible que lorsque le statut de la fertilité des sols est connu. Améliorer la fertilité du sol est très important dans l'augmentation des rendements en riz (Talpur et al., 2013). La forte demande du riz sera comblée grâce à une augmentation de la production à travers une triple augmentation d'application des fertilisants (Cassman et Harwood, 1995 ; Koné et al., 2011). II est donc important d'accroitre l'utilisation efficiente et efficace des intrants dans la riziculture à travers les meilleures gestions des sols (Ying et al., 1998) et une maitrise des dates de semis. Aliou et al., (2014) ont montré que des combinaisons d'engrais (NPK et urée) produisent de meilleures performances agronomiques (croissance en hauteur, production de talles et grains par panicules, rendement paddy et paille) indépendamment des variétés de riz et des sites. L'azote est l'un des éléments majeurs à côté du phosphore et potassium pour la nutrition des plantes. II joue plusieurs rôles dans le développement de la plante. II contribue au développement végétatif de toutes les parties aériennes de la plante, feuilles, tiges et formation des graines d'où sa contribution à l'amélioration du rendement. L'azote produit $1-4 \%$ de matière sèche de la plante et une bonne fourniture de l'azote à la plante stimule le développement racinaire ainsi que l'absorption d'autres nutriments (Brady et Weil, 2002). L'azote en retour accroit le rendement grain en augmentant le taux de variables de rendement tels que le nombre de panicules au mètre carré (Nesgea et al., 2012). L'azote est l'élément fertilisant qui est beaucoup plus limitant à la production agricole, d'où son utilisation en très grandes quantités en agriculture. II est le constituant essentiel des protéines et assure donc la qualité optimale en protéine des cultures. Quelque fois, il est responsable de la pollution environnementale parce que le lessivage des nitrates $\left(\mathrm{NO}_{3}^{-}\right)$peut réduire la qualité de la nappe d'eau phréatique et aussi les émissions azotées peuvent contribuer à l'effet de serre. L'ion nitrate étant très mobile dans le sol, et la riziculture pluviale s'effectuant en période de grande quantité d'eau, il devient donc nécessaire de déterminer le bon moment de l'application d'engrais azoté afin d'assurer une production optimale. Les études menées dans l'optique de trouver des variétés les mieux adaptées à l'écologie africaine, ont conduit à l'obtention des nouvelles variétés du riz NERICA (Nouveau Riz pour l'Afrique). Ces variétés sont issues d'un croisement entre deux variétés ancestrales: la variété asiatique (Oryza sativa) très productive et la variété africaine (Oryza glaberima) très résistant aux conditions écologiques africaines (ADRAO, 2008). Avec l'introduction des nouvelles variétés très productives, le prélèvement des nutriments du sol sera excessif lorsque les fertilisants minéraux ne 
seront pas appliqués ou bien seront appliqués en quantité inadéquate (Moro et al., 2008). On va alors assister à un appauvrissement du sol en éléments nutritifs qui contribue à la réduction du rendement et la productivité du sol. La raison d'un faible rendement est principalement liée aux mauvaises techniques culturales. Le semis du riz à la période propice est important pour un rendement élevé. Les dates de semis propice peuvent varier avec le site et les génotypes (Bashir et al., 2010). Yoshida (1981) et Bruns et Abbas (2006) ont reporté que, pour sa phénologie telle que l'initiation paniculaire, la floraison et la maturité, les plants de riz nécessitent une température particulière. Une analyse des variables climatiques de la localité de Santchou faite par Bourgeon (1979) recommandait une prudence quant au choix de la date de semis. La période de Juillet à Octobre était identifiée comme plage sûre pour la riziculture pluviale. Parmi les contraintes pédologiques majeures dans les basfonds rizicoles, figurent en bonne place à côté de la déficience des éléments nutritifs, la toxicité ferreuse (Brady, 1982; Samaranayake et al., 2005) en période de submersion en eau. La toxicité ferreuse est fortement liée à la fluctuation

\section{MATERIELS ET METHODES}

Description du site d'étude: L'étude a été conduite dans la plaine des Mbo, dans les parcelles expérimentales de l'Institut de la Recherche Agricole pour le Développement (IRAD) dans la localité de Santchou de la région de l'Ouest Cameroun. La zone de l'expérimentation est située à $09^{\circ} 58^{\prime} 31$ 'de longitude Est et à $05^{\circ} 15^{\prime} 32^{\prime \prime}$ de latitude Nord avec une altitude de $711 \mathrm{~m}$. Le climat est de type équatorial avec deux saisons : une longue saison des pluies de mi-mars à mi-novembre et une courte saison sèche de minovembre à mi-mars. La pluviométrie annuelle moyenne est autour de $1800 \mathrm{~mm}$. La température minimale moyenne est $18^{\circ} \mathrm{C}$, maximale moyenne est $27^{\circ} \mathrm{C}$ (Tabi et al., 2013), soit une température moyenne annuelle d'environ $23^{\circ} \mathrm{C}$. Les sols des périmètres rizicoles de Santchou présente en surface une forte teneur en argile $(35 \%)$ et en limon (25\%). Ils ont une structure compacte et une texture argilo limoneuse. La couleur du sol est brun clair ou brunâtre avec des taches noires (oxydes ferromanganiques). Leurs de la nappe d'eau du sol et au faible $\mathrm{pH}$ du sol $(\mathrm{pH}$ $<5$ ) dans les bas-fonds. La remontée d'eau dans le sol est plus influencée par la forte pluviométrie et une faible évapotranspiration. La toxicité ferreuse apparait donc à une certaine période de l'année (Asch et al., 2005) c'est-à-dire la période pendant laquelle la nappe d'eau du sol remonte. La teneur élevée en ions ferreux $\mathrm{Fe}^{2+}$ (toxicité ferreuse) engendre des troubles reproductifs chez le riz (Cherif et al., 2009). Le choix des dates de semis et l'utilisation des engrais chimiques sont des moyens de mitigation de l'effet toxique du fer (Diatta et Sahrawat, 2006). C'est parce que les ions $\mathrm{Fe}^{2+}$ peuvent être fixés par les ions phosphate pouvant être apportés en majorité par les engrais chimiques. Le semis direct avec apport des engrais s'avère efficace car les engrais atténueraient l'effet d'une éventuelle toxicité ferreuse avant la levée des plants. Compte tenu de tout ce qui précède, la présente étude vise à évaluer l'effet des différentes dates de semis et niveaux d'azote sur les variables de croissance et de rendement d'une variété améliorée du riz NERICA L56 dans la plaine des Mbo, Cameroun.

caractéristiques chimiques se présentent comme suit : $\mathrm{pH}$ (eau) 5,0 ce sont donc des sols acides ; matière organique $2,68 \%$; azote total $3 \% ; \mathrm{K}^{+}$échangeable 0,13 meq/100g ; CEC 13,30meq/100g et P (Bray2) 324 ppm (Bourgeon, 1979). On y retrouve donc des sols hydromorphes à pseudogley. La topographie est plane, d'où la stagnation des eaux et leur infiltration lente. Comme conséquence, la nappe phréatique est assez superficielle. Selon les critères d'aptitude décrits par Sys et al. (1991), les caractéristiques pédoclimatiques du site de Santchou montrent qu'il est bien favorable à la riziculture pluviale.

Conduite de l'essai : Les essais ont été conduits suivant un dispositif en split plot où la date de semis était le facteur principal et la dose d'urée, le facteur secondaire comportant trois répétitions. Chaque traitement était appliqué à une parcelle expérimentale de $4 \mathrm{~m}^{2}$ soit $2 \mathrm{~m} \times 2 \mathrm{~m}$ la première année et de $9 \mathrm{~m}^{2}$ l'année suivante. Les allés de $0,5 \mathrm{~m}$ et $1 \mathrm{~m}$ ont été laissés respectivement entre les parcelles et les blocs. 
Chaque parcelle élémentaire était constituée de 72 poquets parmi lesquels les 25 poquets centraux $(5$ lignes $x 5$ poquets) formaient le carré de rendement de $1 \mathrm{~m}^{2}$. Tous les essais ont été conduits en condition d'alimentation hydrique strictement pluviale. Pour chacun des essais, les travaux de préparation de terrain ont consisté en un labour à une profondeur de $20 \mathrm{~cm}$ à l'aide de la houe. La géométrie de semis adoptée était de $25 \mathrm{~cm}$ entre les lignes et $22 \mathrm{~cm}$ entre les poquets d'une même ligne, soit une densité de 180 000 poquets/ha. Les semis ont été effectués directement à raison de cinq grains par poquet. Les plants ont été démariés à deux pieds par poquet en condition humide, avant le tallage. Des désherbages manuels ont été effectués au besoin pour maintenir les plants en situation de non compétition avec les adventices. Le traitement fongique contre les maladies du riz, en particulier la pyriculariose, a été appliqué lorsque le besoin se faisait ressentir. Le fongicide utilisé était un fongicide polyvalent (systémique et de contact) le «Fongistar $72 \%$ WP» en utilisation curative et préventive. Les fertilisations chimiques de fond ont été effectuées conformément aux recommandations locales qui consistent en un épandage d'engrais N-P-K (20-10-10) à la dose de $300 \mathrm{~kg} / \mathrm{ha}$ au semis. Le tableau 1 présente les différentes doses d'engrais appliquées. Les doses d'urée ont été apportées en engrais de couverture à raison de $50 \%$ au tallage et $50 \%$ à l'initiation paniculaire. Les semis ont été décalés de deux semaines soit de 14 jours (tableau 2).

Tableau 1 : Combinaisons d'engrais appliqués

\begin{tabular}{lcccc}
\hline Doses d'engrais & \multicolumn{4}{c}{ Désignation } \\
\cline { 2 - 5 } & Engrais de fond & \multicolumn{2}{c}{ Engrais de couverture } & Formulation \\
& NPK (20 -10-10) & AZOTE (Kg/ha) & UREE 46\% N (Kg/ha) & N P K \\
T1 & 0 & 0 & 0 & $00-00-00$ \\
T2 & 300 & 30 & 65,23 & $90-30-30$ \\
T3 & 300 & 60 & 130,46 & $120-30-30$ \\
T4 & 300 & 90 & 195,69 & $150-30-30$ \\
T5 & 300 & 120 & 260,92 & $180-30-30$ \\
\hline
\end{tabular}

Tableau 2 : Dates de semis retenues

\begin{tabular}{lcccc}
\hline Site & \multicolumn{3}{c}{ Essai 1} & \multicolumn{2}{c}{ Essai 2 } \\
\hline Semis & 1 & 2 & 1 & 2 \\
Date & $25 / 07 / 2017$ & $10 / 08 / 2017$ & $22 / 07 / 2018$ & $08 / 08 / 2017$ \\
\hline
\end{tabular}

Matériels végétatif : La variété améliorée NERICA L56 était exclusivement utilisée à cause de son potentiel de rendement. II s'agit d'une variété interspécifique, issue d'un croisement entre les espèces Oryza glabberima et Oryza sativa (ADRAO, 2008). La semence utilisée provenait de l'IRAD. Le pouvoir germinatif de cette semence était de $95 \%$.

Hauteur des plants : La mesure de la hauteur a été effectuée à la récolte sur six poquets sélectionnés au hasard sur $1 \mathrm{~m}^{2}$ de chaque unité expérimentale (carré de rendement). La hauteur (en $\mathrm{cm}$ ) correspondant à une parcelle est la moyenne des mesures effectuées sur les six poquets depuis la base des plants jusqu'au sommet de la plus longue feuille.

Nombre de talles : il est déterminé par comptage des talles sur les plants des six poquets sélectionnés au hasard du carré de rendement à la récolte. Le nombre de talle correspondant à une parcelle est la moyenne des talles obtenues sur les six poquets. Le nombre de talles moyennes par mètre carré est obtenu en multipliant ce résultat par le nombre de poquets du carré de rendement ayant les plants de riz.

Variables de rendement: Pour tous les essais, la production de la biomasse aérienne des six poquets du carré de rendement a été pesée après battage. Cette biomasse était constituée de talles, de feuilles et de la paille des panicules obtenue après battage. Les grains et la biomasse ont été pré-séchés à l'air avant un séjour final à l'étuve à $65^{\circ} \mathrm{C}$ pendant une durée de 72 heures; ce qui a permis de calculer le rendement grains en kg. ha-1 ajusté à $14 \%$ d'humidité (RDTG) (Yoshida, 1981 ; Djomo et al., 2017) et le rendement paille (RDTP) (en $\mathrm{kg} / \mathrm{ha}$ ) à $0 \%$ d'humidité. Le rendement en grains de riz paddy pour les différents traitements a été calculé en utilisant les équations 1 et 2 (Randrianarison, 2011 ; Djomo et al., 2017) : 
$\mathbf{Y}=\mathbf{P L} / \mathbf{m}^{2} \times$ Pan $/$ plant $\times \mathbf{G} /$ Pan $\times \% \mathbf{F G} \times \mathbf{P M G} \times \mathbf{1 0}^{-7}$

Où $Y=$ rendement $; \mathrm{PL} / \mathrm{m}^{2}=$ nombre de plants par mètre carré $;$ Pan/plant $=$ nombre de panicules par plant $; \mathrm{G} / \mathrm{Pan}=$ nombre total de grains par panicule ;

$\operatorname{RDTG}(14 \%)=\frac{100-\text { THG }}{100-14} \times Y$

A la récolte, on a procédé au comptage sur un mètre carré de chaque unité expérimentale du nombre total de talles (TAL) et de panicules (PANI). Les moyennes

$$
\text { INFERT }=\frac{\text { TAL }- \text { PANI }}{\text { TAL }} \times 100
$$

Ensuite, les grains de paddy des panicules de cinq poquets de chaque unité expérimentale ont été récoltés pour la détermination du Poids de Mille Grains (PMG). Le nombre total d'Epillets (EPIL) et d'Epillets Vides

STERIL $=\frac{\text { EPV }}{\text { EPIL }} \times 100$

$\%$ FG $=100-$ STERIL

Traitement des données : Les analyses de la variance ont été effectuées sur les données de chaque campagne. Les tests ont porté sur l'effet de la date de semis, de la dose d'urée et de l'interaction de ces deux facteurs sur les variables étudiées. Pour ce faire, le dispositif en split plot a été considéré, la date de semis étant le facteur principal et la dose d'urée, le facteur secondaire. Ces analyses ont été effectuées à l'aide du logiciel $\mathrm{R}$ version 3.6.1. Le test de Shapiro-Wilk (Shapiro et Wilk, 1965) a permis de vérifier la distribution normale des données et le test de Bartlett (Bartlett, 1937) a été utilisé pour tester l'égalité des

\section{RESULTATS}

Hauteur: Les résultats de l'analyse de la variance (tableau 3) montrent des différences très hautement significative $(p<0,001)$ entre les différentes doses d'azote pour chaque date de semis en ce qui concerne la hauteur à la récolte pour les deux campagnes. Les plants avaient une hauteur moyenne de $122 \mathrm{~cm}$ avec le premier semis contre $110 \mathrm{~cm}$ avec le deuxième semis en 2017 (tableau 4). Malgré que les plants soient plus courts en 2018 qu'en 2017, les plants les plus hauts ont été toujours produits au premier semis $(111 \mathrm{~cm}$ contre $98 \mathrm{~cm}$ ) (tableau 4). Sur les deux années d'essais, les traitements ayant reçu l'engrais NPK en fond et l'urée
$\% F G=$ pourcentage de grains remplis par panicule ; PMG = Poids de Mille Grains pleins ; $10^{-7}$ unité de conversion.

de ces variables ont permis de calculer l'infertilité des talles (INFERT) comme suit :

(EPV) par panicule a été compté pour cinq panicules choisies de façon aléatoire. Les moyennes de ces variables ont été utilisées pour calculer le taux de stérilité (STERIL) des panicules comme suit :

variances. Lorsque les conditions de normalité et d'homoscédasticité n'étaient pas satisfaites, les transformations logarithmiques et racine carrée ont permis de poursuivre les analyses. Toutes les analyses de variance ont été effectuées au seuil de significativité de $5 \%$. En cas de différence significative, les comparaisons multiples de moyennes ont été réalisées grâce au test de Duncan pour les doses d'urée. Le test de Student a été utilisé pour comparer les moyennes des différentes variables pour les deux dates de semis de chaque essai.

en couverture, ont enregistré des meilleures performances en hauteur comparativement au témoin n'ayant pas reçu d'engrais. Les plants produits avec les traitements T3 (300Kg NPK $20-10-10+60 \mathrm{Kg} \mathrm{N})$, T4 $(300 \mathrm{Kg} \mathrm{NPK} 20-10-10+90 \mathrm{Kg} \mathrm{N})$ et T5 (300Kg NPK $20-10-10+120 \mathrm{Kg} \mathrm{N}$ ) ne différaient statistiquement pas en taille. Nonobstant cette uniformité statistique de hauteur, le traitement T5 aux premiers semis a donné des plants les plus hauts $(124,27 \mathrm{~cm}$ en moyenne) (tableau 4). Par contre, aux deuxièmes semis, T4 a été le traitement qui a donné des meilleures performances $(120,27 \mathrm{~cm}$ en moyenne) (tableau 4). 
Nombre de talles: Le nombre de talles a significativement baissé de la première date de semis à la deuxième sur les deux années d'essais. Cette baisse était de $14 \%$ (244 à 211 talles $\left./ \mathrm{m}^{2}\right)$ en 2017 et de $39 \%$ (228 à 139 talles $/ \mathrm{m}^{2}$ ) en 2018. En prenant séparément les dates de semis, les doses d'azote ont eu d'effets significatifs sur le nombre de talles pour les deux essais. En 2017, la dose $120 \mathrm{Kg} / \mathrm{ha}$ a produit plus de talles (309 talles $/ \mathrm{m}^{2}$ ) comparativement aux autres doses par rapport à la première date de semis. Les doses $90 \mathrm{Kg} / \mathrm{ha}$ et $120 \mathrm{Kg} / \mathrm{ha}$ d'azote ont produit plus de talles au mètre carré. En 2018, les doses d'azote de 60 à $120 \mathrm{Kg} / \mathrm{ha}$ à la première date de semis ont produit plus de talles comparativement à la dose de $30 \mathrm{Kg} / \mathrm{ha}$ et le témoin. Malgré que ces doses produisent des tallages abondant, la dose $120 \mathrm{Kg} / \mathrm{ha}$ s'est démarqué des deux autres en produisant plus de talles (221 et 171 talles $/ \mathrm{m}^{2}$ ) pour les deux dates de semis (tableau 4).

Nombre de panicules: le nombre de panicules a significativement baissé de la première date de semis à la deuxième sur les deux années d'essais. Cette baisse était de $20 \%$ (229 à 184 panicules $\left./ \mathrm{m}^{2}\right)$ en 2017 et de $42 \%$ (196 à 115 panicules $/ \mathrm{m}^{2}$ ) en 2018. Sur les deux années d'essais, le nombre de panicules produites ne différaient pas statistiquement $(p>0,05)$ entre les traitements regroupés par semis. Malgré ce manque de différence entre les traitements, le traitement T5 (dose d'azote de $120 \mathrm{Kg} / \mathrm{ha}$ ) a produit plus de panicule sur les deux années d'essais (241 Panicules/ $\mathrm{m}^{2}$ en moyenne aux premiers semis contre 194 Panicules $/ \mathrm{m}^{2}$ aux deuxièmes semis) (tableau 4).

Infertilité : Le pourcentage de talles infertiles a significativement augmenté de la première à la deuxième date de semis en passant de 6,85 à $15,75 \%$ en 2017 et de 3,02 à $20,34 \%$ en 2018. Les doses d'azote appliquées ont produit moins de talles infertiles sur les deux années d'essais. Globalement, le taux de talles infertiles a été décroissant lorsque la dose d'azote appliquée augmentait (tableaux 3 et 4).

Tableau 3 : Résultats de l'analyse de la variance (valeur de F) sur la hauteur des plants à maturité $(\mathrm{cm})$, le nombre de talles et panicules au mètre carré $\left(\mathrm{m}^{2}\right)$ et l'infertilité $(\%)$ considérant les différentes doses d'engrais appliquées et les dates de semis

\begin{tabular}{|c|c|c|c|c|c|c|c|c|c|}
\hline \multirow{3}{*}{$\begin{array}{l}\text { Sources de } \\
\text { variation }\end{array}$} & \multirow[t]{3}{*}{ Ddl } & \multicolumn{4}{|c|}{ Valeur de F } & \multirow{2}{*}{\multicolumn{2}{|c|}{ Panicule/m² }} & \multirow{2}{*}{\multicolumn{2}{|c|}{ Infertilité (\%) }} \\
\hline & & \multicolumn{2}{|c|}{ Hauteur $(\mathrm{cm})$} & \multicolumn{2}{|c|}{ Talles $/ \mathrm{m}^{2}$} & & & & \\
\hline & & 2017 & 2018 & 2017 & 2018 & 2017 & 2018 & 2017 & 2018 \\
\hline Doses d'engrais & 4 & $49,15^{\star \star \star}$ & $28,99^{* * *}$ & $87,50^{* \star *}$ & $43,27^{\star \star *}$ & $104,99^{* * *}$ & $63,02^{* * *}$ & $16,79^{\star \star *}$ & $9,45^{\star * *}$ \\
\hline Semis & 1 & $39,29^{* * *}$ & $38,47^{* * *}$ & $22,92^{* * *}$ & $94,59^{* * *}$ & $48,49^{* * *}$ & $122,26^{* * *}$ & $25,43^{* * *}$ & $15,90^{* * *}$ \\
\hline $\begin{array}{l}\text { Doses d'engrais } \\
\text { *semis }\end{array}$ & 4 & $3,63^{*}$ & $2,15 \mathrm{~ns}$ & $0,84 \mathrm{~ns}$ & $7,06^{* *}$ & $0,82 \mathrm{~ns}$ & $8,43^{* * *}$ & $7,52^{* * *}$ & $0,39 \mathrm{~ns}$ \\
\hline
\end{tabular}

$\mathrm{ddl}=$ degré de liberté $; \mathrm{ns}=$ non significatif $;{ }^{*}=\mathrm{P}<0,05 ;{ }^{* *}=\mathrm{P}<0,01 ;{ }^{* * *}=\mathrm{P}<0,001$

Tableau 4 : Effet des doses d'engrais et des dates de semis sur la hauteur des plants à maturité $(\mathrm{cm})$, le nombre de talles et panicules au mètre carré $\left(\mathrm{m}^{2}\right)$ et l'infertilité $(\%)$

\begin{tabular}{|c|c|c|c|c|c|c|c|c|c|}
\hline Semis & Doses d'engrais & \multicolumn{2}{|c|}{ Hauteur $(\mathrm{cm})$} & \multicolumn{2}{|c|}{ Talles $/ \mathrm{m}^{2}$} & \multicolumn{2}{|c|}{ Panicule $/ \mathrm{m}^{2}$} & \multicolumn{2}{|c|}{ Infertilité (\%) } \\
\hline \multirow{8}{*}{ Semis 1} & & 2017 & 2018 & 2017 & 2018 & 2017 & 2018 & 2017 & 2018 \\
\hline & T1 & $100,17 d$ & $82,58 \mathrm{c}$ & $114 c$ & $69 \mathrm{c}$ & 101c & $51 \mathrm{c}$ & $11,93 a$ & $26,86 a$ \\
\hline & T2 & $125,28 \mathrm{bc}$ & $102,30 \mathrm{~b}$ & $236 b$ & $172 b$ & $221 b$ & $133 b$ & $6,40 \mathrm{~b}$ & $18,54 b$ \\
\hline & T3 & $121,72 \mathrm{c}$ & $112,30 a$ & $276 a b$ & $226 \mathrm{a}$ & $265 a$ & $197 a$ & $3,77 \mathrm{~b}$ & $13,84 b c$ \\
\hline & $\mathrm{T} 4$ & $129,74 b$ & $110,80 a$ & $287 a b$ & $229 a$ & $275 a$ & $202 a$ & $4,40 \mathrm{~b}$ & $13,01 b c$ \\
\hline & T5 & $135,44 a$ & $113,10 \mathrm{a}$ & $309 a$ & $221 a$ & $285 a$ & $196 \mathrm{a}$ & $7,77 \mathrm{~b}$ & $11,14 \mathrm{c}$ \\
\hline & Moyenne & $122,47 \mathrm{~A}$ & $110,85 \mathrm{~A}$ & $244,1 \mathrm{~A}$ & $228 \mathrm{~A}$ & $229,1 \mathrm{~A}$ & $196 \mathrm{~A}$ & $6,85 \mathrm{~B}$ & $13,02 \mathrm{~B}$ \\
\hline & CV (\%) & 10,58 & 13,92 & 29,82 & 37,58 & 31,60 & 40,44 & 57,32 & 42,95 \\
\hline \multirow{7}{*}{ Semis 2} & $\mathrm{~T} 1$ & $77,94 \mathrm{c}$ & $82,00 \mathrm{~b}$ & $97 \mathrm{c}$ & $73 c$ & $58 d$ & $50 \mathrm{c}$ & $39,52 a$ & $32,08 a$ \\
\hline & T2 & $105,61 b$ & $95,67 a$ & $213 b$ & $115 b c$ & $196 c$ & $92 b c$ & $7,89 b$ & $20,94 a b$ \\
\hline & T3 & $119,94 a$ & $103,8 a$ & $231 a b$ & 168ab & $205 b c$ & $137 a b$ & $11,09 b$ & $18,43 b$ \\
\hline & T4 & $123,44 a$ & $102,8 a$ & $256 a$ & $166 a b$ & $227 a b$ & $145 a$ & $11,02 b$ & $16,72 b$ \\
\hline & $\mathrm{T} 5$ & $121,61 \mathrm{a}$ & $103,9 a$ & $259 a$ & $171 a$ & $235 a$ & $153 a$ & $9,22 b$ & $13,54 b$ \\
\hline & Moyenne & 109,71B & $97,62 \mathrm{~B}$ & $211,20 B$ & 139B & $184,20 \mathrm{~B}$ & $115 B$ & $15,75 \mathrm{~A}$ & $20,34 \mathrm{~A}$ \\
\hline & CV (\%) & 16,94 & 9,77 & 30,36 & 32,69 & 36,95 & 38,10 & 84,34 & 40,75 \\
\hline
\end{tabular}


Les moyennes suivies des mêmes lettres alphabétiques dans une colonne ne sont pas significativement différentes $(P>0,05)$ d'après le test de Duncan ; T1 (0 Kg/ha NPK + 0 Kg/ha N) ; T2 (300Kg NPK 20-10- $10+30 \mathrm{Kg} \mathrm{N}) ;$ T3 (300Kg NPK 20- 10- $10+60$ Kg N) ; T4 (300Kg NPK $20-10-10+90 \mathrm{Kg} \mathrm{N}) ;$ T5 (300Kg NPK 20- $10-10+120 \mathrm{Kg} \mathrm{N})$

Stérilité des panicules: Les résultats de l'analyse de la variance (tableau 5 ) indiquent des différences hautement significatives $(p<0,01)$ à très hautement significatives $(p<0,001)$ entre les doses d'azote appliquée d'une part et les dates de semis d'autre part en ce qui concerne la stérilité des grains de paddy. Par contre, seules les dates de semis ont présenté des différences hautement significatives $(p<0,01)$ en ce qui concerne la variable Poids de Mille Grains PMG. Les résultats (tableau 6) montrent que la stérilité décroît lorsque les doses d'azote augmentent. Les traitements T4 et T5 sont ceux qui produisent moins de paddy vides. Leurs stérilités moyennes sur les deux années d'essai varient entre 8 et $11 \%$.

Tableau 5 : Résultats de l'analyse de la variance (valeur de F) sur le Poids de Mille Grains (PMG), le nombre total de grains et des grains vides (NTG et NTGV) et la stérilité (\%) considérant les différentes doses d'azote appliquées et les dates de semis

\begin{tabular}{|c|c|c|c|c|c|c|c|c|c|}
\hline \multirow{3}{*}{$\begin{array}{l}\text { Sources de } \\
\text { variation }\end{array}$} & \multirow[t]{3}{*}{ ddl } & \multicolumn{8}{|c|}{ Valeur de F } \\
\hline & & \multicolumn{2}{|c|}{ PMG } & \multicolumn{2}{|c|}{ NTG/Pan } & \multicolumn{2}{|c|}{ NTGV/Pan } & \multicolumn{2}{|c|}{ Stérilité } \\
\hline & & 2017 & 2018 & 2017 & 2018 & 2017 & 2018 & 2017 & 2018 \\
\hline Doses d'engrais & 4 & $2,32 \mathrm{~ns}$ & $2,49 n s$ & $18,19^{* * *}$ & $\overline{101,78^{\star \star \star}}$ & $1,26 \mathrm{~ns}$ & $2,24 n s$ & $5,47^{* *}$ & $14,37^{\star \star \star}$ \\
\hline Semis & 1 & 10,08 ** & $76,12^{\star \star *}$ & $0,66 \mathrm{~ns}$ & $0,23 \mathrm{~ns}$ & $29,47^{* * *}$ & $18,92^{* * *}$ & $53,08 * * *$ & $21,80^{\star * *}$ \\
\hline $\begin{array}{l}\text { Doses d'engrais } \\
\text { *semis }\end{array}$ & 4 & 0,22 ns & $15,02^{* * *}$ & $4,78^{* *}$ & $23,31^{* * *}$ & $2,22 \mathrm{~ns}$ & $3,66^{*}$ & $1,09 \mathrm{~ns}$ & $0,54 \mathrm{~ns}$ \\
\hline
\end{tabular}

Tableau 6 : Effet des doses d'engrais et des dates de semis sur le poids de mille grains(PMG), le nombre total de grains et des grains vides (NTG et NTGV) et la stérilité (\%)

\begin{tabular}{|c|c|c|c|c|c|c|c|c|c|}
\hline \multirow[t]{2}{*}{ Semis } & \multirow[t]{2}{*}{ Doses d'engrais } & \multicolumn{2}{|c|}{ PMG } & \multicolumn{2}{|c|}{ NTG/Pan } & \multicolumn{2}{|c|}{ NTGV/Pan } & \multicolumn{2}{|c|}{ Stérilité } \\
\hline & & 2017 & 2018 & 2017 & 2018 & 2017 & 2018 & 2017 & 2018 \\
\hline \multirow{7}{*}{ Semis 1} & T1 & $24,56 a$ & $25,26 b$ & $122 \mathrm{c}$ & $107 d$ & $22 a$ & $26 a b$ & $17,75 a$ & $25,72 a$ \\
\hline & T2 & $27,98 a$ & $26,06 a$ & $132 b c$ & $165 c$ & $17 a$ & $26 a b$ & $12,79 a b$ & $15,40 \mathrm{~b}$ \\
\hline & T3 & $25,96 \mathrm{a}$ & $25,59 a b$ & $166 a$ & $179 b$ & $21 \mathrm{a}$ & $27 a$ & $12,35 a b$ & $14,76 \mathrm{bc}$ \\
\hline & T4 & $25,87 a$ & $25,41 b$ & $151 \mathrm{abc}$ & $190 a$ & $13 a$ & $21 b$ & $8,40 \mathrm{~b}$ & $11,05 \mathrm{bc}$ \\
\hline & T5 & $26,57 a$ & $25,32 b$ & $155 a b$ & $192 a$ & $17 a$ & $19 b$ & $10,77 \mathrm{~b}$ & $9,65 c$ \\
\hline & Moyenne & $26,19 \mathrm{~A}$ & $26,32 \mathrm{~A}$ & $145,27 \mathrm{~A}$ & $168 \mathrm{~A}$ & $17,87 \mathrm{~B}$ & $19 \mathrm{~B}$ & $12,41 \mathrm{~B}$ & $11,85 B$ \\
\hline & CV $(\%)$ & 8,33 & 2,51 & 14,63 & 12,28 & 34,31 & 35,65 & 34,87 & 45,00 \\
\hline \multirow{7}{*}{ Semis 2} & $\mathrm{~T} 1$ & $23,03 a$ & $23,77 \mathrm{c}$ & $70 c$ & $77 c$ & $23 b$ & $23 a$ & $33,70 a$ & $30,76 a$ \\
\hline & $\mathrm{T} 2$ & $25,65 a$ & $26,23 a$ & $152 a b$ & $174 b$ & $32 a b$ & $31 a$ & $21,20 b$ & $17,82 b$ \\
\hline & T3 & $24,70 \mathrm{a}$ & $25,38 \mathrm{~b}$ & $180 a$ & $189 a$ & $40 a$ & $36 a$ & $22,34 b$ & $18,89 \mathrm{~b}$ \\
\hline & $\mathrm{T} 4$ & $23,01 \mathrm{a}$ & $23,76 c$ & $158 a b$ & $196 a$ & $38 a b$ & $29 a$ & $24,17 a b$ & $14,76 \mathrm{~b}$ \\
\hline & T5 & $23,96 \mathrm{a}$ & $24,53 \mathrm{c}$ & $142 b$ & $195 a$ & $28 a b$ & $23 a$ & $19,56 b$ & $11,72 \mathrm{c}$ \\
\hline & Moyenne & $24,07 \mathrm{~B}$ & $24,75 \mathrm{~B}$ & $140,42 \mathrm{~A}$ & $166 \mathrm{~A}$ & $32,39 \mathrm{~A}$ & $28,26 \mathrm{~A}$ & $24,19 \mathrm{~A}$ & $18,79 \mathrm{~A}$ \\
\hline & CV $(\%)$ & 6,46 & 4,14 & 29,30 & 27,62 & 29,69 & 25,62 & 28,05 & 41,11 \\
\hline
\end{tabular}

Les moyennes suivies des mêmes lettres alphabétiques de même caractère dans une colonne ne sont pas significativement différentes $(P>0,05)$ d'après le test de Duncan ;T1 $(0 \mathrm{Kg} / \mathrm{ha} \mathrm{NPK}+0 \mathrm{Kg} / \mathrm{ha} \mathrm{N}) ; \mathrm{T} 2(300 \mathrm{Kg} \mathrm{NPK} \mathrm{20-10-10+30} \mathrm{Kg} \mathrm{N}) ; \mathrm{T} 3$ (300Kg NPK 20- 10- $10+60 \mathrm{Kg} \mathrm{N})$; T4 (300Kg NPK 20-10- $10+90 \mathrm{Kg} \mathrm{N}) ;$ T5 (300Kg NPK 20- 10- $10+120 \mathrm{Kg} \mathrm{N})$

PMG : Poids mille grains ; NTG : Nombre Total de Grains ; PAN : panicule ; NTGV : Nombre Total de Grains Vides

Rendements grains de riz paddy et paille: Les doses d'azote et les dates de semis présentent des différences hautement significatives $(p<0,001)$ (tableau 9) en ce qui concerne les rendements grains et paille.
Les interactions entre les doses d'azote et dates de semis, en ce qui concerne les rendements grains et paille sont significatives, sauf au premier semis 2017 pour le rendement paille $(p>0,05)$. Globalement, les 
rendements (grains et paille) étaient croissant avec les doses d'azote et décroissants avec les dates de semis sur les deux essais. Les rendements en grains pour les traitements ayant reçu l'engrais variaient de 7,50 à $10,82 \mathrm{t} / \mathrm{ha}$ au premier semis et de 5,26 à $6,23 \mathrm{t} / \mathrm{ha}$ au deuxième semis de l'essai de 2017. Soit une diminution d'environ 2t/ha du premier au deuxième semis. En 2018, les variations étaient de 5,04 à 9,14 t/ha au premier semis et de 3,63 à 6,47 t/ha au deuxième semis: soit une diminution d'environ 2 t/ha. Les rendements en paille variaient de 4,55 à 6,39 t/ha au premier semis et de 3,82 à 5,96 au deuxième semis en 2017 : soit une diminution d'environ 1 t/ha. En 2018, les variations étaient de 5,21 à 8,92 tha au premier semis et de 2,86 à 5,88 t/ha au deuxième semis: soit une diminution d'environ 3 tha. Les doses d'azote ont été plus rentables à la première date de semis qu'à la seconde. Les rendements (grains et paille) ne différaient pas statistiquement entre les traitements T3 (300Kg NPK 20- 10- $10+60 \mathrm{Kg} \mathrm{N})$, T4 (300Kg NPK 20- $10-10+90 \mathrm{Kg} \mathrm{N})$ et T5 (300Kg NPK 20- 10- $10+$ $120 \mathrm{KgN})$ sur les deux années d'essai et par date de semis. La riziculture pluviale sans fertilisation n'est pas recommandable dans un tel environnement. II ressort du tableau 10 que, les rendements (grains et paille) du témoin (T1) étaient pratiquement 4 fois plus faibles que ceux des traitements ayant reçu les engrais. Dans l'ensemble des deux essais, les variations étaient de 2,49 à 0,55 tha (2017) et de 1,12 à 0,77 tha (2018). On note que la performance en ce qui concerne le traitement témoin $\mathrm{T} 1$ est bonne en premiers semis qu'aux deuxièmes semis.

Tableau 9 : Résultats de l'analyse de la variance (valeur de F) sur le rendement (grains et pailles) et l'indice de récolte considérant les différentes doses d'engrais appliquées et les dates de semis

\begin{tabular}{lccccc}
\hline Sources de variation & ddl & \multicolumn{4}{c}{ Valeur de F } \\
\cline { 3 - 6 } & & \multicolumn{2}{c}{$\begin{array}{c}\text { Rendement Grains } \\
\text { (t/ha) }\end{array}$} & \multicolumn{2}{c}{$\begin{array}{c}\text { Rendement Paille } \\
\text { (t/ha) }\end{array}$} \\
\hline & & 2017 & 2018 & 2017 & 2018 \\
\cline { 3 - 6 } Doses d'engrais & 4 & $56,35^{* * *}$ & $63,36^{* * *}$ & $25,50^{* * *}$ & $87,21^{* * *}$ \\
Semis & 1 & $104,28^{* * *}$ & $106,59^{* * *}$ & $15,51^{* * *}$ & $293,42^{* * *}$ \\
Doses d'engrais semis & 4 & $3,22^{*}$ & $6,35^{* *}$ & $0,52 \mathrm{~ns}$ & $22,09^{* * *}$ \\
\hline
\end{tabular}

$\mathrm{Ddl}=$ degré de liberté $; \mathrm{ns}=$ non significatif $;{ }^{*}=\mathrm{P}<0,05 ;{ }^{* *}=\mathrm{P}<0,01 ;{ }^{* \star *}=\mathrm{P}<0,001$

Tableau 10: Effet des doses d'engrais et des dates de semis sur le rendement (grains et pailles) et l'indice de récolte

\begin{tabular}{|c|c|c|c|c|c|}
\hline \multirow[t]{2}{*}{ Semis } & \multirow[t]{2}{*}{ Doses d'engrais } & \multicolumn{2}{|c|}{$\begin{array}{c}\text { Rendement Grains } \\
\text { (t/ha) }\end{array}$} & \multicolumn{2}{|c|}{$\begin{array}{c}\text { Rendement Paille } \\
\text { (t/ha) }\end{array}$} \\
\hline & & 2017 & 2018 & 2017 & 2018 \\
\hline & T1 & $2,49 c$ & $1,12 \mathrm{c}$ & $1,96 \mathrm{c}$ & $1,31 \mathrm{c}$ \\
\hline & T2 & $7,50 \mathrm{~b}$ & $5,04 b$ & $4,55 b$ & $5,21 \mathrm{~b}$ \\
\hline \multirow[t]{7}{*}{ Semis 1} & T3 & $9,70 a b$ & $7,93 a$ & $5,42 a b$ & $7,88 a$ \\
\hline & T4 & $9,88 a b$ & $8,68 a$ & $6,39 a$ & $8,55 a$ \\
\hline & T5 & $10,82 a$ & $9,14 a$ & $6,59 a$ & $8,92 \mathrm{a}$ \\
\hline & Moyenne & $8,07 \mathrm{~A}$ & $8,32 \mathrm{~A}$ & $4,98 \mathrm{~A}$ & $8,99 \mathrm{~A}$ \\
\hline & CV $(\%)$ & 39,06 & 47,91 & 37,31 & 47,91 \\
\hline & $\mathrm{T} 1$ & $0,55 b$ & $0,77 c$ & $0,85 b$ & $\overline{1,23 d}$ \\
\hline & $\mathrm{T} 2$ & $5,26 a$ & $3,63 b$ & $3,82 a$ & $2,86 \mathrm{c}$ \\
\hline \multirow[t]{5}{*}{ Semis 2} & T3 & $6,23 a$ & $5,43 a b$ & $4,16 a$ & $4,73 \mathrm{~b}$ \\
\hline & T4 & $5,55 a$ & $5,98 a$ & $5,36 \mathrm{a}$ & $4,12 \mathrm{~b}$ \\
\hline & T5 & $5,73 a$ & $6,47 a$ & $4,52 \mathrm{a}$ & $5,88 a$ \\
\hline & Moyenne & $4,66 \mathrm{~B}$ & $4,44 \mathrm{~B}$ & $3,74 \mathrm{~A}$ & $3,76 \mathrm{~B}$ \\
\hline & CV (\%) & 50,38 & 51,54 & 47,42 & 43,89 \\
\hline
\end{tabular}

Les moyennes suivies des mêmes lettres alphabétiques de même caractère dans une colonne ne sont pas significativement différentes $(P>$ $0,05)$ d'après le test de Duncan; T1 (0 Kg/ha NPK + $0 \mathrm{Kg} / \mathrm{ha} \mathrm{N}) ; \mathrm{T} 2(300 \mathrm{Kg} \mathrm{NPK} 20-10-10+30 \mathrm{Kg} \mathrm{N}) ; \mathrm{T} 3(300 \mathrm{Kg} \mathrm{NPK} 20-10-10+60 \mathrm{Kg}$ N) ; T4 (300Kg NPK 20- 10- $10+90 \mathrm{Kg} \mathrm{N})$; T5 (300Kg NPK 20- 10- $10+120 \mathrm{Kg} \mathrm{N})$ 


\section{DISCUSSION}

Les études des différentes doses d'engrais sur les variables de croissante et de rendement ont montré que la hauteur augmentait avec la dose d'engrais. La dose $120 \mathrm{Kg} / \mathrm{ha}$ d'azote a enregistré la meilleure hauteur au premier semis. Le résultat était similaire au deuxième semis mais avec une diminution en hauteur des plants. Ces différences en taille peuvent être attribuées à la capacité du NL56 à répondre à l'accroissement de la dose d'azote. Gala et al. (2011) ont montré que l'augmentation des quantités d'azote améliore considérablement la croissance végétative du riz. Ces résultats sont en accord avec ceux de Khakwari et al. (2006) et Akram et al. (2007). Ils ont montré que la hauteur des plants de riz était significativement affectée par les dates de semis et que le semis précoce (en Juillet) du riz produisait des plants les plus hauts comparés à ceux du semis tardif (en Août). Le nombre de talles augmentait significativement avec la dose d'engrais quelle que soit la date de semis. Cet effet est plus important pour le semis précoce que le semis tardif. L'augmentation de la dose d'engrais riche en azote influence le nombre de talles (Sanogo et al., 2010). Le stress hydrique serait peut-être le facteur justifiant cette différence entre le nombre de talles des deux dates de semis. La période d'application de l'azote est donc primordiale pour son utilisation efficiente par la plante. Le résultat est identique quant au nombre de panicules car le nombre de talles et panicules sont étroitement liés. Le stress hydrique durant le tallage réduit considérablement le nombre de talles (Mohamed, 2005 ; Aliou et al., 2014). Les doses élevées d'azote réduisent le nombre de talles infertiles pour les deux semis. Cette infertilité est plus élevée au deuxième semis. Ceci peut être expliqué par le fait que la formation de talles et panicules dépend de la température de croissance du riz $\left(13^{\circ} \mathrm{C}\right)$ (Kouakou et al., 2016). Plus il pleut, plus l'humidité atmosphérique est basse. Ce qui favorise le développement abondant des talles et par conséquent des panicules. Les résultats indiquent que le premier semis a produit plus de talles fertiles que le semis tardif. Le riz produit moins de talles fertiles et de panicules en conditions de stress hydrique à la phase reproductive et d'inondation excessive à la phase végétative (Kouakou, 2016). Dans la zone d'étude, ces phénomènes sont susceptibles de survenir pour un semis en Août (semis tardif). L'infertilité est le facteur important dans le choix d'une date optimale de semis car le nombre de panicules est un indicateur du meilleur rendement grain du riz. Ces résultats sont similaires à ceux d'Osman et al. (2015).
Ils ont montré que pour une différence d'au moins 14 jours entre les dates de semis, le nombre de talles fertiles a été statistiquement différent. Les doses d'engrais n'ont pas eu d'effet significatif sur le poids de 1000 grains de riz paddy. Seules les dates de semis ont eu d'effet significatif. Les grains les plus lourds sont produits au premier semis. Les conditions climatiques (pluviométrie et température) sont les seuls facteurs explicatifs de cette différence. Plus ces conditions sont meilleures, plus les grains de riz paddy produits sont lourds. Ces résultats sont similaires à ceux d'Osman et al. (2015). Ils ont démontré qu'une différence entre les dates de semis d'au moins deux semaines a un effet significatif sur le Poids de Mille Grains de riz. Le NTG croit avec les doses d'engrais, mais n'est pas influencé par les dates de semis. Les doses élevées d'azote produisaient plus de grains de paddy. Le NTGV et la stérilité diminuaient significativement lorsque les doses d'engrais augmentaient. Pour une même dose d'azote, le NTGV et la stérilité augmentaient du semis précoce au semis tardif. Le stress hydrique dû à la baisse de la pluviométrie au semis tardif serait à l'origine de cette différence. Les résultats similaires ont été obtenus par Osman et al. (2015) qui ont prouvé qu'à des dates de semis différentes, la stérilité augmente du semis précoce au semis tardif. Les rendements grains et pailles ont été significativement affectés par les doses d'engrais et les dates de semis. Les rendements augmentent avec les doses d'engrais et diminuent avec les dates de semis. Ces résultats peuvent être expliqués par le faite que le rendement en grains de riz est fortement influencé par les paramètres tels que: nombre de panicule, de grains par panicule, de grains vides par panicule et Poids de Mille Grains qui dépendent d'engrais et les dates de semis. Sanogo et al. (2010) ont reporté que le rendement en grain augmente lorsque la fertilisation du riz NERICA est adéquate. Les résultats similaires ont été obtenus par Osman et al. (2015) qui ont montré que les rendements élevés pourraient être obtenus lorsque la date de semis est optimale. Les meilleures performances sont obtenues pour le semis à la troisième décade du mois de Juillet avec la dose d'engrais $(300 \mathrm{Kg} / \mathrm{ha}$ de NPK 20-10-10 et $120 \mathrm{Kg}$ d'azote sous forme d'urée). Une explication plausible de ces observations est le fait qu'une forte pluviométrie occasionnerait la toxicité ferreuse dans un bas-fond. La mitigation de l'effet toxique du fer ferreux passerait par le choix adéquat de la date de semis et l'utilisation d'une combinaison d'engrais à la dose adéquate (Asch et al., 2005). Il est 
évident que les doses d'azote et les dates de semis sont de facteurs important à prendre en compte dans la

\section{CONCLUSION}

L'application des différentes doses d'engrais chimiques (NPK 20-10-10 et Urée) en riziculture pluviale de basfond dans la plaine des Mbo (Santchou) donne une meilleure compréhension de l'importance de la fertilisation raisonnée. Les traitements ayant reçu les fertilisants chimiques (NPK en engrais de fond et l'urée en engrais de couverture) ont tous induit de meilleures performances agronomiques dépendamment des dates de semis. Ces performances sont croissantes avec les doses d'azote et décroissantes lorsque les dates de semis retardent. Les résultats de cette recherche ont montré qu'une meilleure valorisation des engrais était intrinsèquement liée à la date de semis. Ainsi, sur les

\section{REMERCIEMENTS}

Les auteurs remercient le chef d'antenne de l'IRAD de Santchou d'avoir offert les parcelles expérimentales et

\section{REFERENCES BIBLIOGRAPHIQUES}

ADRAO (Centre du riz pour l'Afrique). 2008. Guide pratique de la culture des NERICA de plateau 2008. Cotonou, Bénin : Centre du riz pour l'Afrique (ADRAO). 36 pp.

Akram, H.M., Ali, A., Nadeem, lqbal, M.S.2007. Yield and yield components of rice varieties as affected by transplanting dates. Journal of Agricultural Research (Pakistan) 45, 105-111.

Aliou, S., Denis, G.K., Ibouraïman, B., Romain, H.S., Valentin, K.M., 2014. Effet de l'urée et du NPK 15-15-15 perlés et super granulés sur la productivité des variétés de riz IR841 et NERICA-L14 en zone de bas-fond au SudBénin. Journal of Applied Biosciences 77, 6575.

Asch, F., Becker, M., Kpongor, D.S., 2005. A quick and efficient screen for resistance to iron toxicity in lowland rice. J. Plant Nutr. Soil Sci. 168, 764773.

Bartlett, M. S. 1937. "Properties of sufficiency and statistical tests". Proceedings of the Royal Statistical Society, Series A 160, 268-282

Bashir, M.U., Akbar, N., Iqbal, A., Zaman, H., 2010. Effect of different sowing dates on yield and yield. Pak. J. Agri. Sci 47, 361-365.

Bourgeon G., 1979. Aménagement de la Plaine des MBOS. Etude morphologique de détail.

Brady, N.C. et Weil, R. R., 2002. Nature and Properties recherche d'une production optimale du riz paddy dans les hautes terres de l'ouest Cameroun.

deux dates de semis, les doses de 90 à $120 \mathrm{~kg} / \mathrm{ha}$ d'azote (en couverture) ont été beaucoup plus performantes pour les variables mesurées comparativement aux doses de moins de $90 \mathrm{Kg} / \mathrm{ha}$. II serait donc souhaitable d'utiliser la formulation NPK: 180 - 30 - 30, au mois de Juillet (à la troisième décade qui est la période de semis optimale) pour la riziculture pluviale de NERICA L56 en semis direct dans la plaine des Mbo.: II convient également de mettre l'accent sur les bonnes pratiques agricoles afin d'assurer une utilisation efficace et effective des nutriments par la plante.

l'appui technique pour la réalisation de cette recherche ayant abouti à la rédaction de cet article.

of Soils, 13th Edition.

Cassman, K.G., Harwood, R.R., 1995. The nature of agricultural systems: food security and environmental balance. Food Policy, Getting Agriculture Moving 20, 439-454.

Chérif, M., Audebert, A., Fofana, M., Zouzou, M., n.d. Evaluation of Iron Toxicity on Lowland Irrigated Rice in West Africa 5.

Diatta, S., Sahrawat, L.K., 2006. La toxicité ferreuse du riz en afrique de l'ouest. criblage de variétés tolérantes et rôle de N, P, K ET ZN, Centre du riz pour l'Afrique (ADRAO). ed. A. Audebert, L.T. Narteh, P. Kiepe, D. Millar et B. Beks, 01 BP 2031 Cotonou, Bénin.

Djomo S.H., Mbong G. A., Malla D. K., Suh C., 2017. Effect of different doses of NPK fertilizer on the growth and yield of rice in Ndop, North West of Cameroon. AJAR 12, 1244-1252.

Gala, J.B.T., Maméri, C., Albert, Y.-K., Jules, K.Z., 2011. Rentabilité des engrais minéraux en riziculture pluviale de plateau: Cas de la zone de Gagnoa dans le centre ouest de la Côte d'Ivoire 10.

Goufo, P., 2008. Rice Production in Cameroon: a Review 12.

Jones, D.T.1995.Protein Secondary Structure Prediction Based on Position-specific Scoring Matrices 8. 
Khakwani, A.A., M. Zubair, Mansoor, M., Khalid Naveed, Shah, I.H., Wahab, A., Ilyas, M., Ahmed, I., 2006. Agronomic and Morphological Parameters of Rice Crop as Affected by Date of Transplanting. Journal of Agronomy 5, 248-250.

Koné, B., Amadji, G.L., Aliou, S., Diatta, S., Akakpo, C., 2011. Nutrient constraint and yield potential of rice on upland soil in the south of the Dahoumey gap of West Africa. Archives of Agronomy and Soil Science 57, 763-774.

Kouakou, K.P.M., Muller, B., Fofana, A., Guisse, A., 2016. Performances agronomiques de quatre variétés de riz pluvial NERICA de plateau semées à différentes dates en zone soudanosahélienne au Sénégal. Journal of Applied Biosciences 99, 9382.

Macauley, H., Ramadjita, T., 2015. Les cultures céréalières riz maïs millet sorgho et blé. Africa Rice

MINADER, 2012. Annuaire des Statistiques du Secteur Agricole. Campagnes 2009 et 2010 (No. 17). Cameroun.

Molua, E.L., 2010. Rice Production Response to Trade Liberalization in Cameroon 12.

Moro, B.M., Nuhu, I.R. and Toshiyuki, W. 2008. Determining optimum rates of mineral fertilizers for economic rice grain yields under the "Sawah" System in Ghana. West African Journal of Applied Ecology 12: 1-12.

Nesgea, S., Gebrekidan, H., Sharma, J.J., Berhe, T., 2012. Effects of Nitrogen and Phosphorus Fertilizer Application on Yield Attributes, Grain Yield and Quality of Rain Fed Rice (NERICA3) in Gambella, South western Ethiopia. East African Journal of Sciences Volume 6, 91-104.

Osman, K.A., Mustafa, A.M., Elsheikh, Y.M.A., Idris, A.E., 2015. Influence of different sowing dates on growth and yield of direct seeded rice (Oryza sativa L.) in semi-arid zone (Sudan). International Journal of Agronomy and Agricultural Research (IJAAR) 6, 38-48.

Osman, K.T., 2013. Soils: principles, properties and management. Springer, Dordrecht ; New York.

Randrianarison L., 2011. Contribution à l'étude économique de la fertilisation du sol sur culture de riz en sri : cas de l'utilisation du compost tananamadio et du taroka dans la commune rurale de behenjy. Mémoire d'ingénieur Agronome université d'Antananarivo $66 \mathrm{p}$.

Samaranayake, P., Peiris, B.D., Dssanayake, S., 2005. Effect of Excessive Ferrous (Fe2+) on Growth and Iron Content in Rice (Oryza sativa)

Sanogo S., Camara M., Zouzou M., Sekou A., 2010. Effets de la fertilisation minérale sur des variétés améliorées de riz en condition irriguée à Gagnoa, Côte d'Ivoire 9 .

Seck, P.A., Togola, A., Touré, A., Diagne, A., 2013. Propositions for optimizing the performance of rice production in West Africa. Cahiers Agricultures 22, 361-368.

Shapiro S. S. and M. B. Wilk, " An analysis of variance test for normality (complete samples) ", Biometrika, vol. 52, no 3-4, 1965, p. 591-611

Sys, C., Van Ranst, E., Debaveye, J., 1991. Land Evaluation PART I: Principles in Land Evaluation and Crop Production Calculations, Agricultural Publications. Nouvelle Imprimerie Duculot S.A., Gembloux (Belgique), Place du Champ de Mars 5 nte 57-1050 Brussels Belgium.

Tabi, F.O., Ngobesing, E.S.C., Yinda, G.S., Boukong, A., Omoko, M., Bitondo, D., Mvondo, Z.A.D., 2013. Soil fertility capability classification (FCC) for rice production in Cameroon lowlands. African Journal of Agricultural Research 8, 1650-1660.

Talpur, A.D., Ikhwanuddin, M., Ambok Bolong, A.-M., 2013. Nutritional effects of ginger (Zingiber officinale Roscoe) on immune response of Asian sea bass, Lates calcarifer (Bloch) and disease resistance against Vibrio harveyi. Aquaculture 400-401, 46-52.

Vanlauwe, B., Palm, C.A., Murwira, H.K., Merckx, R., 2002. Organic resource management in subSaharan Africa: validation of a residue qualitydriven decision support system. Agronomie 22,839-846.

Yoshida S. 1981. Fundamentals of rice crop science. International Rice Research Institute, Los Banos, Laguna, Philippines 94-110. 